\title{
Benchmarking Nonequilibrium Green's Functions against Configuration Interaction for time-dependent Auger decay processes
}

\author{
F. Covito, ${ }^{1}$ E. Perfetto, ${ }^{2,3}$ A. Rubio, ${ }^{1,4,5}$ and G. Stefanucci ${ }^{3,6}$ \\ ${ }^{1}$ Max Planck Institute for the Structure and Dynamics of Matter and Center for Free-Electron Laser Science, \\ Luruper Chaussee 149, 22761 Hamburg, Germany \\ ${ }^{2}$ CNR-ISM, Division of Ultrafast Processes in Materials (FLASHit), \\ Area della ricerca di Roma 1, Monterotondo Scalo, Italy \\ ${ }^{3}$ Dipartimento di Fisica, Università di Roma Tor Vergata, \\ Via della Ricerca Scientifica, 00133 Rome, Italy \\ ${ }^{4}$ Center for Computational Quantum Physics (CCQ), \\ The Flatiron Institute, 162 Fifth avenue, New York NY 10010 \\ ${ }^{5}$ Nano-Bio Spectroscopy Group, Universidad del País Vasco, 20018 San Sebastin, Spain \\ ${ }^{6}$ INFN, Sezione di Roma Tor Vergata, Via della Ricerca Scientifica 1, 00133 Roma, Italy
}

(Dated: October 4, 2018)

\begin{abstract}
We have recently proposed a Nonequilibrium Green's Function (NEGF) approach to include Auger decay processes in the ultrafast charge dynamics of photoionized molecules. Within the so called Generalized Kadanoff-Baym Ansatz the fundamental unknowns of the NEGF equations are the reduced one-particle density matrix of bound electrons and the occupations of the continuum states. Both unknowns are one-time functions like the density in Time-Dependent Functional Theory (TDDFT). In this work we assess the accuracy of the approach against Configuration Interaction (CI) calculations in one-dimensional model systems. Our results show that NEGF correctly captures qualitative and quantitative features of the relaxation dynamics provided that the energy of the Auger electron is much larger than the Coulomb repulsion between two holes in the valence shells. For the accuracy of the results dynamical electron-electron correlations or, equivalently, memory effects play a pivotal role. The combination of our NEGF approach with the Sham-Schlüter equation may provide useful insights for the development of TDDFT exchange-correlation potentials with a history dependence.
\end{abstract}

\section{INTRODUCTION}

Photo-ionized many-body systems relax to lower energy states through nuclear rearrangement and charge redistribution. Nuclear dynamics does typically play a role on longer time scales, although there are situations where electron-nuclear and electron-electron interactions compete on the same timescale, e.g., in the vicinity of a conical intersection. At the (sub)femtosecond timescale, however, the most relevant relaxation channel of coreionized molecules is the Auger decay which is exclusively driven by the Coulomb interaction [1].

Recent advances in pump-probe experiments made it possible to follow the attosecond dynamics of atoms after the sudden expulsion of a core electron $2[6]$. Theoretical frameworks describing the Auger decay have been proposed, the more accurate being the ones based on manybody wavefunctions, see also Ref. [7. Although these methods are in principle applicable to atoms as well as molecules, they quickly become prohibitive for systems with more than a few active electrons. For instance, Auger decays in ionized small molecules or molecules of biological interest are extremely difficult to cope with wavefunction approaches due to the large number of states involved in the process. Still, Auger decays contribute to the relaxation dynamics of these more complex systems, which are currently attracting an increasing interest and attention [8] 12. It is therefore crucial to develop first-principles approaches capable of capturing the (sub)femtosecond relaxation mechanisms induced by electronic correlations and applicable to atoms as well as molecules.

The most widely used method for large scale real-time simulations is Time-Dependent Density Functional Theory 13 15] (TDDFT), which gives an adequate and computationally affordable tool for the description of systems consisting of up to thousands of atoms. The most efficient and extensively used functionals for TDDFT calculations are the space-time local exchange-correlation (xc) functionals. It has been shown numerically in Ref. [16] that these approximate functionals fail in capturing Auger decays, the fundamental reason being that they lack memory effects - the xc potential depends on the instantaneous density only.

We have recently proposed a first-principles NonEquilibrium Green's Function (NEGF) approach 17 which overcomes the limitation of adiabatic functionals and that may inspire new ideas for the inclusion of memory effects in the TDDFT functionals. The method is applicable to molecules with up to tens of atoms and at its core there is an equation to simulate the electron dynamics in the parent cation without dealing explicitly with the Auger electrons. The idea is similar in spirit to the embedding scheme in time-dependent quantum transport where the electron dynamics in the molecular junction is simulated without dealing explicitly with the electrons in the leads [18 22]. However, whereas in quantum transport the integration out of electrons in the leads gives 
an embedding self-energy which is independent of the density in the junction, the integration out of the Auger electrons gives an Auger self-energy which is a functional of the density in the molecule.

In order to assess the quality of the NEGF approach in this work we use the time-dependent charge distribution of the bound electrons to reconstruct the Auger wavepacket in free space, and then benchmark the results against exact configuration interaction (CI) calculations. We perform NEGF and CI simulations in a model onedimensional (1D) system and study the real space-time shape of the Auger wavepacket as well as the Auger spectrum. The main outcome of this investigation is that the results of the NEGF approach are in excellent agreement with those from CI provided that the repulsion between the valence holes is much smaller than the energy of the Auger electron.

\section{DESCRIPTION OF THE SYSTEM AND THEORY}

Let us consider a 1D finite system described by the oneparticle Hartree-Fock (HF) basis $\left\{\varphi_{i}, \varphi_{\mu}\right\}$, where roman indices run over bound states and greek indices run over continuum states. The equilibrium Hamiltonian can be conveniently written as the sum of three terms

$$
\hat{H}^{\mathrm{eq}}=\hat{H}_{\text {bound }}+\hat{H}_{\text {Auger }}+\hat{H}_{\text {cont }},
$$

where $\hat{H}_{\text {bound }}$ is the bound electrons Hamiltonian, $\hat{H}_{\text {Auger }}$ is the Auger interaction and $\hat{H}_{\text {cont }}$ is the free-continuum part. In our basis, these are written as

$$
\begin{aligned}
& \hat{H}_{\text {bound }}=\sum_{i j} h_{i j} \hat{c}_{i}^{\dagger} \hat{c}_{j}+\frac{1}{2} \sum_{i j m n} v_{i j m n} \hat{c}_{i}^{\dagger} \hat{c}_{j}^{\dagger} \hat{c}_{m} \hat{c}_{n}, \\
& \hat{H}_{\text {Auger }}=\sum_{i j m} \sum_{\mu}\left(v_{i j m \mu}^{A} \hat{c}_{i}^{\dagger} \hat{c}_{j}^{\dagger} \hat{c}_{m} \hat{c}_{\mu}+\text { H.c. }\right), \\
& \hat{H}_{\text {cont }}=\sum_{\mu} \epsilon_{\mu} \hat{c}_{\mu}^{\dagger} \hat{c}_{\mu},
\end{aligned}
$$

where $c_{i}^{\dagger}\left(c_{i}\right)$ is the creation (annihilation) operator for the state $\varphi_{i}$ (the same convention applies to the continuum index $\mu), h_{i j}$ are the one-electron integrals, $\epsilon_{\mu}$ are the continuum single-particle HF energies and $v_{i j m n}$ $\left(v_{i j m \mu}^{A}\right)$ are the two-electron Coulomb integrals responsible for intra-molecular (Auger) scatterings. The oneand two-electron integrals are defined as

$$
\begin{aligned}
h_{i j} & \equiv \int d x \varphi_{i}^{\star}(x)\left[-\frac{1}{2} \nabla_{x}^{2}+V_{n}(x)\right] \varphi_{j}(x), \\
v_{i j m n} & \equiv \int d x d x^{\prime} \varphi_{i}^{\star}(x) \varphi_{j}^{\star}\left(x^{\prime}\right) V_{e}\left(x, x^{\prime}\right) \varphi_{m}\left(x^{\prime}\right) \varphi_{n}(x),
\end{aligned}
$$

with $V_{n}(x)$ and $V_{e}\left(x, x^{\prime}\right)$ the nuclear and electronelectron potential. Note that the Auger Coulomb integrals $v_{i j m \mu}^{A}$ are defined according to Eq. 3b with $n=\mu$.
In Eq. (1) we discard all the off-diagonal contribution $h_{i \mu}$, $h_{\mu \mu^{\prime}}$ as well as all Coulomb integrals with more than one index in the continuum. This approximation does not affect the physical description of the dynamics as demonstated by comparisons against full grid calculations in Ref. [17. In fact, in the HF basis both $h_{i \mu}$ and $h_{\mu \mu^{\prime}}$ are much smaller than $h_{i j}$ and $\epsilon_{\mu}$ whereas Coulomb integrals with two or more indices in the continuum are responsible for scattering process that are highly suppressed by phase-space arguments if the photoelectron energy is much larger than the kinetic energy of the Auger electron. Henceforth, this condition is assumed to be fulfilled.

The explicit simulation of the ionization process with a laser field does not represent a complication for the NEGF method. In fact, the general framework presented in Ref. 17] accounts for the coupling of external fields with the bound-bound and bound-continuum dipole matrix elements. Instead, the framework discards the coupling of external fields with the continuumcontinuum dipole matrix elements and, therefore, lightfield streaking experiments relevant to, e.g., attosecond metrology 23], or multuphoton ionization processes are left out.

In this work we focus on the dynamics induced by the sudden removal of a core electron, thus the ionization process is not simulated. An additional simplification used for the simulations below (which is however not essential for the approach) consists in keeping only integrals of the form $v_{c \mu v_{1} v_{2}}^{A}$, where $c$ labels the state of the suddenly created core hole, $v_{1}$ and $v_{2}$ label two valence states and $\mu$ an arbitrary continuum state. We also observe that the HF wavefunctions are real since the Hamiltonian is invariant under time-reversal. This implies that the Coulomb integrals have the following symmetries

$$
v_{i j m n}=v_{j i n m}=v_{i m j n}=v_{n j m i}
$$

and the like with $n \rightarrow \mu$.

\section{A. NEGF equations}

The derivation of the NEGF equations within the so called Generalized Kadanoff-Baym Ansatz [24] (GKBA) has been presented elsewhere [17; here we only describe the structure of these equations without entering into the complex mathematical and numerical details.

Let $\rho$ be the one-particle reduced density matrix in the bound sector and $f_{\mu}$ be the occupations of the continuum states. Then the NEGF equations read

$$
\left\{\begin{array}{l}
\dot{\rho}=-i\left[h_{\mathrm{HF}}[\rho], \rho\right]-\mathcal{I}[\rho, f]-\mathcal{I}^{\dagger}[\rho, f] \\
\dot{f}_{\mu}=-\mathcal{J}_{\mu}[\rho, f]-\mathcal{J}_{\mu}^{*}[\rho, f]
\end{array},\right.
$$

where the single-particle HF Hamiltonian is defined according to

$$
h_{\mathrm{HF}, i j}=h_{i j}+\sum_{m n}\left(v_{i m n j}-v_{i m j n}\right) \rho_{n m} .
$$


The matrix $\mathcal{I}$ and the scalar $\mathcal{J}_{\mu}$ at time $t$ are explicit functionals of $\rho$ and $f$ at all previous times. They are evaluated using the so-called second-Born (2B) approximation which has been shown to contain the fundamental scattering of the Auger process [25, 26]. The dependence on $\rho$ and $f$ occurs through the lesser and greater GKBA Green's functions 24

$$
G^{\lessgtr}(t, \bar{t})=\mp\left[G^{\mathrm{R}}\left(t, t^{\prime}\right) \rho^{\lessgtr}\left(t^{\prime}\right)-\rho^{\lessgtr}(t) G^{\mathrm{A}}\left(t, t^{\prime}\right)\right],
$$

and the like for $G \lessgtr$ with indices in the continuum. Here, the retarded $\left(G^{\mathrm{R}}\right)$ and advanced $\left(G^{\mathrm{A}}\right)$ Green's functions are evaluated in the HF approximation (and hence they are functionals of $\rho$ and $f$ too). The functional $\mathcal{I}\left(\mathcal{J}_{\mu}\right)$ is linear in $G^{\lessgtr}$ with indices in the continuum and quartic (cubic) in $G \lessgtr$ with indices in the bound sector. Their calculation requires to perform an integral from some initial time, say $t=0$, up to time $t$. The implementation of Eqs. (5) does therefore scale quadratically with the number of time steps. Notice that by setting $\mathcal{I}=\mathcal{J}_{\mu}=0$ is equivalent to perform time-dependent $\mathrm{HF}$ simulations. Like the adiabatic approximations in TDDFT, HF is local in time and therefore it is unable to describe Auger decays.

The scaling of the calculation of $\mathcal{I}$ and $\mathcal{J}_{\mu} \quad$ with the number of basis functions is $\max \left[\left(N_{\text {bound }}\right)^{\mathfrak{p}},\left(N_{\text {bound }}\right)^{\mathfrak{q}} N_{\text {cont }}\right]$, where $N_{\text {bound }}$ is the number of bound states, $N_{\text {cont }}$ the number of continuum states and the exponents $3 \leq \mathfrak{p} \leq 5,2 \leq \mathfrak{q} \leq 4$ depend on the number of nonvanishing Coulomb integrals [17. Currently, both $\mathcal{I}$ and $\mathcal{J}_{\mu}$ are implemented in the CHEERS code [27] which, for $\mathcal{J}_{\mu}=0$, has been recently used to study the charge transfer dynamics in a donor- $\mathrm{C}_{60}$ model dyad 28] and the ultrafast charge migration in the phenylalanine aminoacid up to $40 \mathrm{fs}$ [29. Since the calculation of $\mathcal{J}_{\mu}$ is not heavier than the calculation of $\mathcal{I}$, the NEGF approach can be used to study time-dependent Auger processes driven by XUV or X-ray pulses in molecules with up to tens of atoms.

\section{B. CI calculation}

Let us consider the simplest possible case of a system with one occupied core state, one occupied valence state and a continuum of empty states. We are interested in describing the evolution of the system starting from the initial state

$$
\left|\phi_{x}\right\rangle=c_{c \uparrow}^{\dagger} c_{v \downarrow}^{\dagger} c_{v \uparrow}^{\dagger}|0\rangle
$$

representing a core-hole of down spin. The evolution operator defined by the Hamiltonian in Eq. (1) mixes $\left|\phi_{x}\right\rangle$ with (we recall that only Coulomb integrals of the form $v_{c \mu v v}$ and the like related by symmetries are nonvanish- ing, see Section II

$$
\begin{aligned}
& \left|\phi_{g}\right\rangle=c_{c \uparrow}^{\dagger} c_{c \downarrow}^{\dagger} c_{v \uparrow}^{\dagger}|0\rangle, \\
& \left|\phi_{\mu}\right\rangle=c_{c \uparrow}^{\dagger} c_{c \downarrow}^{\dagger} c_{\mu \uparrow}^{\dagger}|0\rangle,
\end{aligned}
$$

where $\left|\phi_{g}\right\rangle$ is the "intermediate" state with the filled core, i.e., the ground state of the parent cation, and $\left|\phi_{\mu}\right\rangle$ is the state describing the dication with an Auger electron in the continuum state $\mu$. Carrying out the calculations it is easy to show that these states are coupled by the Hamitonian as follows

$$
\begin{aligned}
\hat{H}^{\mathrm{eq}}\left|\phi_{x}\right\rangle & =E_{x}\left|\phi_{x}\right\rangle+T\left|\phi_{g}\right\rangle+\sum_{\mu} V_{\mu}\left|\phi_{\mu}\right\rangle, \\
\hat{H}^{\mathrm{eq}}\left|\phi_{g}\right\rangle & =E_{g}\left|\phi_{g}\right\rangle+T\left|\phi_{x}\right\rangle, \\
\hat{H}^{\mathrm{eq}}\left|\phi_{\mu}\right\rangle & =E_{\mu}\left|\phi_{x}\right\rangle+V_{\mu}\left|\phi_{x}\right\rangle
\end{aligned}
$$

where the energies $E_{x}, E_{g}, E_{\mu}, T$ and $V_{\mu}$ are given by

$$
\begin{aligned}
& E_{x}=h_{c c}+2 h_{v v}+2 v_{c v v c}+v_{v v v v}-v_{c v c v}, \\
& E_{g}=2 h_{c c}+h_{v v}+2 v_{c v v c}+v_{c c c c}-v_{c v c v}, \\
& E_{\mu}=2 h_{c c}+\epsilon_{\mu}+v_{c c c c} \\
& T=h_{c v}+v_{c c v c}+v_{c v v v} \\
& V_{\mu}=v_{v v c \mu} .
\end{aligned}
$$

The simplification brought about by the HF basis is now evident. The HF Hamiltonian $h_{\mathrm{HF}, i j}=h_{i j}+$ $\sum_{k}^{\text {occ }}\left(2 v_{i k k j}-v_{i k j k}\right)$ is diagonal in the HF basis, therefore

$$
\begin{aligned}
0=h_{\mathrm{HF}, c v} & =h_{c v}+\left(2 v_{c c c v}-v_{c c v c}\right)+\left(2 v_{c v v v}-v_{c v v v}\right) \\
& =h_{c v}+v_{c c c v}+v_{c v v v} \equiv T .
\end{aligned}
$$

Thus the "intermediate" state $\left|\phi_{g}\right\rangle$ decouples from the dynamics.

We write the three-body wave function at time $t$ as

$$
|\psi(t)\rangle=a_{x}(t)\left|\phi_{x}\right\rangle+\sum_{\mu} a_{\mu}(t)\left|\phi_{\mu}\right\rangle,
$$

with initial condition $|\psi(0)\rangle=\left|\phi_{x}\right\rangle$. Taking into account Eqs. 10, the time-dependent Schrödinger equation yields a set of coupled equations for the coefficients of the expansion

$$
\left\{\begin{array}{l}
i \dot{a}_{x}(t)=E_{x} a_{x}(t)+\sum_{\mu} V_{\mu} a_{\mu}(t) \\
i \dot{a}_{\mu}(t)=V_{\mu} a_{x}(t)+E_{\mu} a_{\mu}(t)
\end{array}\right.
$$

to be solved with boundary conditions $a_{x}(0)=1$ and $a_{k}(0)=0$.

From the definitions in Eqs. 11 it follows that for the continuum three-body state to have the same energy of the initial state, i.e., $E_{\mu}=E_{x}$, the energy $\epsilon_{\mu}$ of the Auger electron has to be

$$
\epsilon_{\mu} \equiv \epsilon_{\text {Auger }}^{\mathrm{CI}}=2 \epsilon_{v}^{\mathrm{HF}}-\epsilon_{c}^{\mathrm{HF}}-v_{v v v v},
$$


where

$$
\begin{aligned}
& \epsilon_{c}^{\mathrm{HF}}=h_{c c}+v_{c c c c}+2 v_{c v v c}-v_{c v c v} \\
& \epsilon_{v}^{\mathrm{HF}}=h_{v v}+v_{v v v v}+2 v_{v c c v}+v_{v c v c}
\end{aligned}
$$

are the core and valence $\mathrm{HF}$ energies, respectively. It is therefore reasonable to expect a peak in the continuum occupations $f_{\mu}$ for the $\mu$ corresponding to an energy close to the value in Eq. 15 .

In the next Section we solve numerically Eqs. (14). However, in order to get some physical insight into the solution we here make a "wide-band-limit approximation" (WBLA) and carry on the analytic treatment a bit further. Integrating the second equation (14) we have

$$
a_{\mu}(t)=-i \int_{0}^{t} d t^{\prime} e^{-i E_{\mu}\left(t-t^{\prime}\right)} V_{\mu} a_{x}\left(t^{\prime}\right),
$$

which correctly satisfies the boundary conditions $a_{\mu}(0)=$ 0 . Substituing this result into the first equation (14) we get

$$
i \dot{a}_{x}(t)=E_{x} a_{x}(t)+\int_{0}^{\infty} d t^{\prime} K\left(t-t^{\prime}\right) a_{x}\left(t^{\prime}\right)
$$

where

$$
\begin{aligned}
K\left(t-t^{\prime}\right) & =-i \theta\left(t-t^{\prime}\right) \sum_{\mu} V_{\mu}^{2} e^{-i E_{\mu}\left(t-t^{\prime}\right)} \\
& \equiv \int \frac{d \omega}{2 \pi} e^{-i \omega\left(t-t^{\prime}\right)}\left[\Lambda(\omega)-\frac{i}{2} \Gamma(\omega)\right]
\end{aligned}
$$

and

$$
\Lambda(\omega)-\frac{i}{2} \Gamma(\omega)=\sum_{\mu} \frac{V_{\mu}^{2}}{\omega-E_{\mu}+i 0^{+}} .
$$

The real function $\Lambda$ is connected to $\Gamma$ through a Hilbert transform, i.e.,

$$
\Lambda(\omega)=\int \frac{d \omega^{\prime}}{2 \pi} \frac{\Gamma\left(\omega^{\prime}\right)}{\omega-\omega^{\prime}},
$$

and from Eq. (20) it is easy to show that

$$
\Gamma(\omega)=2 \pi \sum_{\mu} V_{\mu}^{2} \delta\left(\omega-E_{\mu}\right) .
$$

For systems in a box of lenght $L$ the continuum wavefunctions are proportional to $1 / \sqrt{L}$ and hence $V_{\mu}^{2}$ scales like $1 / L$, see definition in Eq. (3b). In the limit $L \rightarrow \infty$ the discrete sum in Eq. 22 becomes an integral and $\Gamma(\omega)$ becomes a smooth function of $\omega$. Assuming that $E_{x}$ is a few times larger than $\Gamma\left(E_{x}\right)$ and that $\Gamma(\omega)$ is a slowly varying function for $\omega \simeq E_{x}$, we can then neglect the frequency dependence in $\Gamma$ :

$$
\Gamma(\omega) \simeq \Gamma\left(E_{x}\right) \equiv \gamma
$$

which implies, see Eq. (21), that we can approximate $\Lambda \simeq$ 0 , see Eq. (21). This is the so called WBLA, according to which the kernel $K$ in Eq. 19 can be approximated as

$$
K\left(t-t^{\prime}\right)=-\frac{i}{2} \gamma \delta\left(t-t^{\prime}\right)
$$

Substituing this result into Eq. 18 and then using Eq. (17) it is straighforward to find the following analytic solution

$$
\begin{aligned}
& a_{x}(t)=e^{-i E_{x} t-\frac{\gamma}{2} t}, \\
& a_{\mu}(t)=-V_{\mu} \frac{e^{-i\left(E_{x}-\frac{i}{2} \gamma\right) t}-e^{-i E_{\mu} t}}{E_{\mu}-E_{x}+\frac{i}{2} \gamma} .
\end{aligned}
$$

From Eqs. 25 we infer that the occupation of the continuum states is peaked at $E_{\mu}=E_{x}$ or, equivalently, at $\epsilon_{\mu}=\epsilon_{\text {Auger }}^{\text {CI }}$, in agreement with the discussion above Eq. 15. We emphasize that this conclusion is based on the WBLA. The exact solution contains a small correction which is proportional to the Hilbert transform of $\Gamma(\omega)$ at frequency $\omega \simeq E_{x}$.

\section{Comparing NEGF with CI}

In the NEGF approach at the 2B level of approximation two holes, in addition to feel an average (HF) potential generated by all other electrons, scatter directly once. However, for a strong enough repulsion $v_{v v v v}$ it is necessary to include multiple valence-valence scatterings to predict the correct energy of the Auger electron. In fact, the red shift $v_{v v v v}$ in Eq. 115 can be captured only by summing multiple scatterings to infinite order (T-matrix approximation) 30, 31. Since the 2B approximation includes just a single scattering, the predicted Auger energy is

$$
\epsilon_{\text {Auger }}^{2 \mathrm{~B}}=2 \epsilon_{v}^{\mathrm{HF}}-\epsilon_{c}^{\mathrm{HF}} .
$$

In $3 \mathrm{D}$ molecules the neglect of $v_{v v v v}$ has only a minor impact on the internal (bound-electrons) dynamics since $v_{v v v v}$ is typically less than $1 \mathrm{eV}$ and $\Gamma(\omega)$ varies rather slowly on this energy scales. In this work, however, we are also interested in the description of the Auger wavepacket. Taking into account that the repulsion $v_{v v v v}$ in $1 \mathrm{D}$ systems is larger than in $3 \mathrm{D}$ ones, a sizable difference between the $\mathrm{CI}$ and $2 \mathrm{~B}$ results has to be expected. To demonstrate that such a difference does not affect the overall physical picture nor the details of the Auger wavepacket but only the speed at which the Auger electron is expelled, we isolate the effects of multiple valencevalence scatterings from the CI formulation. Let us express the energy $E_{x}$ defined in Eq. 16a in terms of HF energies

$$
E_{x}=2 \epsilon_{v}^{\mathrm{HF}}+\epsilon_{c}^{\mathrm{HF}}-v_{c c c c}-4 v_{v c c v}+2 v_{v c v c}-v_{v v v v} .
$$

The HF energy $\epsilon_{v}^{\mathrm{HF}}$ is blue shifted by $v_{v v v v}$, see Eq. 16 , an effect captured by the $2 \mathrm{~B}$ approximation. The effect 
of multiple scatterings manifests in the red shift given by the last term of Eq. (27). In the next Section we show that solving Eqs. (14) using for $E_{x}$ the value in Eq. 27) with $v_{v v v v}=0$ one recovers the NEGF results (notice that this is not equivalent to set $v_{v v v v}=0$ in the Hamiltonian since this Coulomb integral renormalizes the HF energy $\epsilon_{v}^{\mathrm{HF}}$ ). We will refer to this CI approximation as CI2B.

\section{RESULTS}

We consider a one-dimensional (1D) atom with soft Coulomb interactions. This particular example is a severe test for the NEGF method since the continuum spectrum has a strong frequency dependence and the valencevalence repulsion energy is of the same order of magnitude of the Auger energy.

The $1 \mathrm{D}$ atom is defined on the points $x_{n}=n a$ of a 1D grid, with $|n| \leq N_{\text {grid }} / 2$. In our model the Coulomb interaction is different from zero only in a box of radius $R$ centered around the nucleus. The one-body Hamiltonian on the grid reads

$$
h\left(x_{n}, x_{m}\right)=\delta_{n, m}\left[2 \kappa+V_{n}\left(x_{n}\right)\right]-\delta_{|n-m|, 1} \kappa
$$

with $V_{n}(x)=U_{\text {en }} / \sqrt{x^{2}+a^{2}}$ the nuclear potential and $\kappa$ the hopping integral between neighbouring points. Electrons interact through $v\left(x, x^{\prime}\right)=Z U_{\text {ee }} / \sqrt{\left(x-x^{\prime}\right)^{2}+a^{2}}$. We analyze the system using $N_{\text {grid }}=1601$ grid-points and choose the parameters according to (atomic units are used throughout): $a=0.5, \kappa=2, Z=4, U_{\text {en }}=2$, $U_{\text {ee }}=U_{\text {en }} / 2$ and $R=10 a$. With four electrons the HF spectrum has five bound states (per spin), the lowest two of which are occupied. The energies of the occupied levels are $\epsilon_{c}^{\mathrm{HF}}=-4.33$ and $\epsilon_{v}^{\mathrm{HF}}=-1.65$ for the core and valence respectively, yielding a 2B Auger energy $\epsilon_{\text {Auger }}^{2 \mathrm{~B}}=1.02$. We work in the sudden creation approximation, according to which the system is perturbed by suddenly removing a core electron. In the NEGF approach this is simulated by subtracting to the equilibrium density matrix $\rho_{i j}^{\text {eq }}$ an infinitesimal amount of charge from the core, hence $\rho_{i j}(0)=\rho_{i j}^{\mathrm{eq}}-\delta_{i c} \delta_{j c} n_{h}$. In the results below the hole density $n_{h}=0.04$.

Subsequently to the creation of the core hole, the Auger process starts taking place, triggering an internal electron dynamics (refilling of the core state) and the expulsion of charge toward the continuum states. The time-dependent occupation of the core state $n_{c}(t)$ is predicted in both $\mathrm{CI}$ and $2 \mathrm{~B}$ calculations to have the following behavior $n_{c}(t)=1-n_{h} e^{-\Gamma t}$, where $n_{h}$ is the core hole created and $\Gamma$ is the inverse lifetime of the Auger decay. Due to the neglect of multiple scatterings, the Auger decay is faster in $2 \mathrm{~B}$ and the corresponding $\Gamma$ is overestimated by a factor 1.5. As already pointed out, this discrepancy is expected to be much smaller in $3 \mathrm{D}$ molecules since the valence-valence repulsion is not as large.

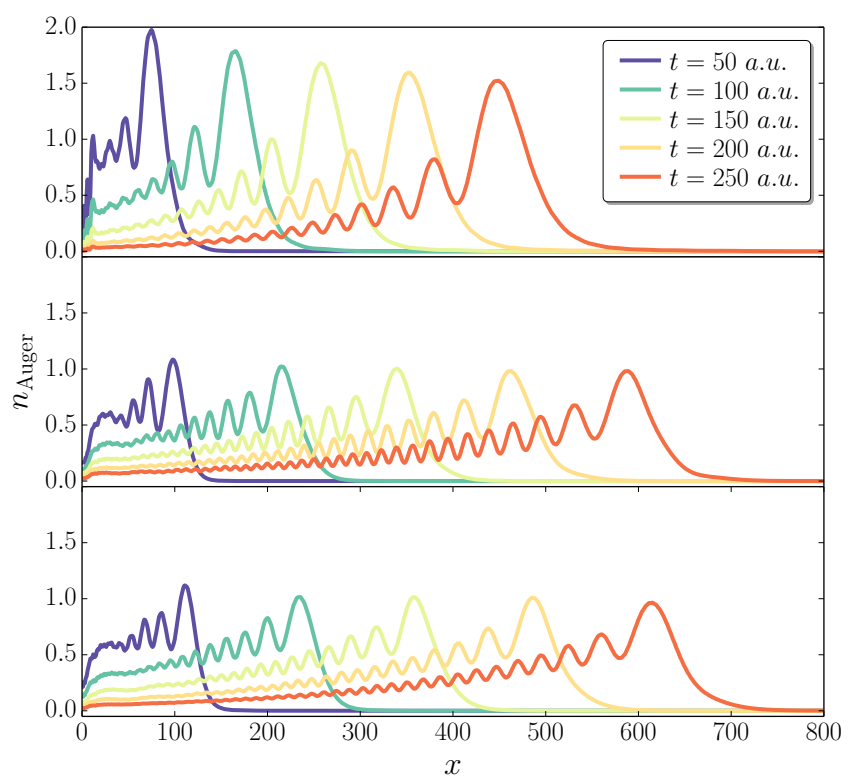

FIG. 1: Snapshots of the density of the Auger wavepacket leaving the atom (nucleus is situated in $x=0$ ) calculated using CI (top), NEGF approach (middle) and CI2B (bottom). The vertical axes have been rescaled by a factor $10^{4}$ for all curves.

In Fig. 1 we display snapshots at different times of the real-space density of the Auger wavepacket as obtained by performing CI (top), NEGF (middle) and CI2B calculations (bottom). The results in the NEGF approach closely resemble the ones in the CI2B treatment, in agreement with the discussion in Section II C. The CI calculation, as expected, shows a slower wavepacket. However, the overall shape, i.e., asymmetric packet with superimposed accumulating ripples on the tail, is common to all methods. We mention that the amplitude of the ripples as well as the wavefront of the Auger wavepacket change if, instead of the sudden creation of a core-hole, we would have simulated the ionization process using an external laser pulse. In fact, these features are not universal and depend on the intensity and duration of the perturbing field [17]. On the other hand, the time $T_{r}$ elapsing between two consecutive maxima at any fixed position is an intrinsic feature of the Auger decay, following the law

$$
T_{r}=\frac{2 \pi}{\epsilon_{\text {Auger }}} .
$$

In the top panel of Fig. 2 we show the time-dependent density $n_{\text {Auger }}\left(x_{0}, t\right)$ of the Auger wavepacket at a certain distance $x_{0}$ from the nucleus. The densities exhibit ripples of different frequency since the energy of the Auger electron is different in CI, NEGF and CI2B. The small discrepancy between NEGF and CI2B is due to the fact that the solution in Eqs. 25 is valid only in the WBLA. Taking into account the frequency dependence of $\Gamma$ one would find a small correction to $E_{\mu}-E_{x}$ proportional to the Hilbert transform of $\Gamma$. From the top panel of Fig. 2 

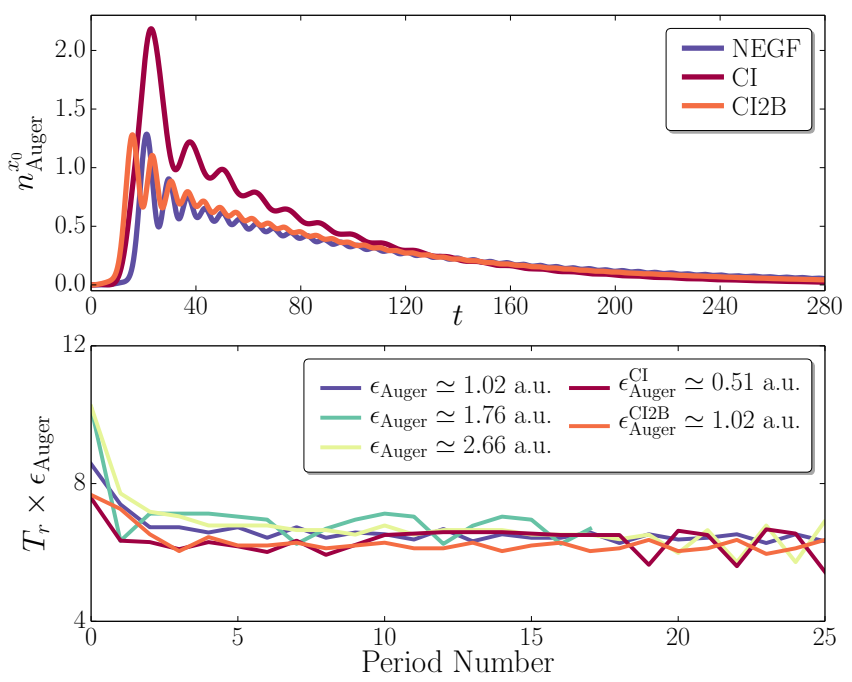

FIG. 2: The top panel shows the time-dependent density of the Auger wavepacket at a fixed distance $x_{0}=30$ from the nucleus for NEGF, CI and CI2B. The bottom panel displays the period of the ripples at $x_{0}$ versus the number of elapsing periods for the three calculations of the top panel and for two more NEGF calculations, see main text.

we see that this correction is rather small and therefore the WBLA is an excellent approximation in this case.

In the bottom panel of Fig. 2 we show the value of the time $T_{r}$ elapsing between two consecutive maxima of the wavepacket versus the number of maxima (counted starting from the left most maximum in the top panel). In the figure $T_{r}$ is rescaled by the Auger energy. In all cases, after a short transient phase, $T_{r}$ attains the value $2 \pi$. In addition to the values of $T_{r}$ corresponding to the three curves of the top panel, in the bottom panel we also report the trend of $T_{r}$ calculated in Ref. 17] for two more NEGF simulations. More specifically, we considered two different combinations of range and strengths of the Coulomb interactions $\left(R, U_{\mathrm{en}}, U_{\mathrm{ee}}\right)=(100 a, 2.6,2.08),(10 a, 2.7,2.025)$ yielding Auger electrons at energies $\epsilon_{\text {Auger }}^{2 \mathrm{~B}}=1.76,2.66$ respectively. As we can see, the quantity $T_{r} \times \epsilon_{\text {Auger }}$ remains independent of the system.

Finally, in Fig. 3 we display the snapshots of the timedependent occupations $f_{\mu}(t)$ of the continuum states $\varphi_{\mu}$. After the creation of the core-hole, occurring at $t=0$, the continuum states start to get populated and, as time passes, gradually get peaked around the Auger energy $\epsilon_{\text {Auger }}^{\mathrm{CI}} \simeq 0.51$ for the CI calculation and $\epsilon_{\text {Auger }}^{2 \mathrm{~B}} \simeq 1$ for the NEGF and CI2B calculation - the small deviation between these two calculations has been discussed previously.

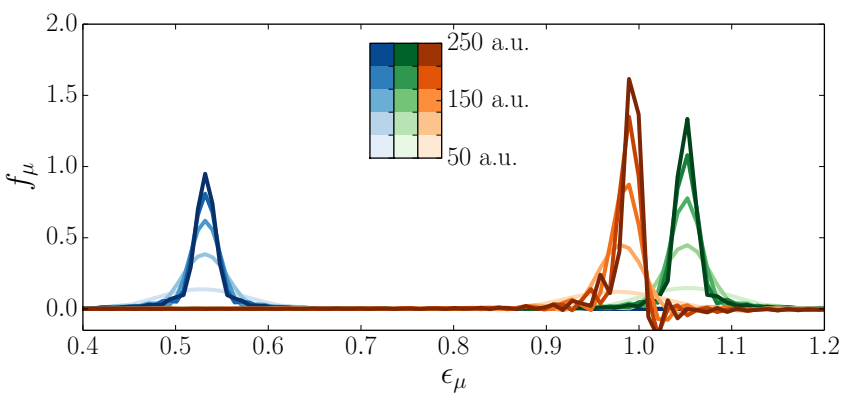

FIG. 3: Snapshots of the occupations $f_{\mu}$ of the continuum states versus their energy $\epsilon_{\mu}$ for CI (blue), CI2B (green) and NEGF (orange). The times of the snapshots (from light to dark) are given by the color bars.

\section{CONCLUSIONS}

To summarize, we have benchmarked a recently proposed NEGF approach [17] against configuration interaction calculations in a simple $1 \mathrm{D}$ model atom. With the exception of the quantitative discrepancies due to the neglect of multiple valence-valence scatterings, good agreement is found for the qualitative features of the Auger process. In fact, NEGF correctly predicts an exponential law for the core-hole refilling and an asymmetric shape of the Auger wavepacket characterized by a long tail with superimposed ripples of period $T_{r}=2 \pi / \epsilon_{\text {Auger }}$. The quantitative difference is only related to the red shift of the energy of the Auger electron, as demonstrated by the agreement between NEGF and CI2B results. We point out that for the systems that we are interested to study in the future, i.e., organic molecules and molecules of biological interest, the valence-valence repulsion is less than $1 \mathrm{eV}$; therefore the neglect of multiple scatterings for the description of the internal dynamics is expected to be less relevant.

The NEGF equations (5) are equations of motion for the one-particle density matrix in the bound sector and for the occupations of the continuum states, not for the Green's function. Both quantities are one-time functions like the charge density of TDDFT $n(\mathbf{r}, t)$. In particular, in a real space basis $\rho(\mathbf{r}, \mathbf{r}, t)=n(\mathbf{r}, t)$. Given the tight relation between $\rho$ and $n$ it would be interesting to use the explicit form of the functionals $\mathcal{I}[\rho, f]$ and $J_{\mu}[\rho, f]$ as a guide to generate approximate xc TDDFT potentials with memory. One possibility would be to combine the linearized Sham-Schlüter equation [32, 33] with NEGF using the Generalized Kadanoff-Baym Ansatz [24].

Akcknowledgements G.S. and E.P. acknowledge EC funding through the RISE Co-ExAN (Grant No. GA644076). E.P. also acknowledges funding from the European Union project $\mathrm{MaX}$ Materials design at the eXascale H2020-EINFRA-2015-1, Grant Agreement No. 676598 and Nanoscience Foundries and Fine AnalysisEurope H2020-INFRAIA-2014-2015, Grant Agreement No. 654360. F.C and A.R. acknowledge financial sup- 
port from the European Research Council (ERC-2015AdG-694097), Grupos Consolidados (IT578-13) and Eu- ropean Union Horizon 2020 program under Grant Agreement 676580 (NOMAD).
[1] R. Pazourek, S. Nagele, and J. Burgdörfer, Rev. Mod. Phys. 87, 765 (2015), URL https://link.aps.org/doi/ 10.1103/RevModPhys.87.765

[2] M. Uiberacker, T. Uphues, M. Schultze, A. J. Verhoef, V. Yakovlev, M. F. Kling, J. Rauschenberger, N. M. Kabachnik, H. Schröder, M. Lezius, et al., Nature 446, 627 (2007).

[3] T. Uphues, M. Schultze, M. F. Kling, M. Uiberacker, S. Hendel, U. Heinzmann, N. M. Kabachnik, and M. Drescher, New Journal of Physics 10, 025009 (2008), URL http://stacks.iop.org/1367-2630/10/i= $2 / a=025009$

[4] M. Drescher, M. Hentschel, R. Kienberger, M. Uiberacker, V. Yakovlev, A. Scrinzi, T. Westerwalbesloh, U. Kleineberg, U. Heinzmann, and F. Krausz, Nature 419, 803 (2002), article, URL http://dx.doi.org/10. 1038 /nature 01143

[5] S. Zherebtsov, A. Wirth, T. Uphues, I. Znakovskaya, O. Herrwerth, J. Gagnon, M. Korbman, V. S. Yakovlev, M. Vrakking, M. Drescher, et al., Journal of Physics B: Atomic, Molecular and Optical Physics 44, 105601 (2011).

[6] J. M. Schins, P. Breger, P. Agostini, R. C. Constantinescu, H. G. Muller, G. Grillon, A. Antonetti, and A. Mysyrowicz, Phys. Rev. Lett. 73, 2180 (1994), URL https://link.aps.org/doi/10. 1103/PhysRevLett.73.2180

[7] A. K. Kazansky, I. P. Sazhina, and N. M. Kabachnik, Journal of Physics B: Atomic, Molecular and Optical Physics 44, 215601 (2011), URL http://stacks.iop. org $/ 0953-4075 / 44 / i=21 / a=215601$.

[8] F. Krausz and M. Ivanov, Rev. Mod. Phys. 81, 163 (2009), URL https://link.aps.org/doi/10.1103/ RevModPhys.81.163.

[9] F. Gao and O. Inganas, Phys. Chem. Chem. Phys. 16, 20291 (2014)

[10] P. Song, Y. Li, F. Ma, T. Pullerits, and M. Sun, The Chemical Record 16, 734 (2016), ISSN 1528-0691, URL http://dx.doi.org/10.1002/tcr.201500244

[11] C. A. Rozzi, F. Troiani, and I. Tavernelli, J. Phys.: Condens. Matter 30, 013002 (2018), URL http://stacks. iop.org/0953-8984/30/i=1/a=013002.

[12] M. Nisoli, P. Decleva, F. Calegari, A. Palacios, and F. Martín, Chemical Reviews 117, 10760 (2017), URL http://dx.doi.org/10.1021/acs.chemrev.6b00453

[13] E. Runge and E. K. U. Gross, Phys. Rev. Lett. 52, 997 (1984), URL https://link.aps.org/doi/10.1103/ PhysRevLett.52.997

[14] C. Ullich, Time-Dependent Density-Functional Theory (Oxford University Press, Oxford, 2012).

[15] N. T. Maitra, The Journal of Chemical Physics 144, 220901 (2016), URL https://doi .org/10.1063/1. 4953039

[16] C. S. Cucinotta, D. Hughes, and P. Ballone, Phys. Rev.
B 86, 045114 (2012), URL https://link.aps.org/doi/ 10.1103/PhysRevB.86.045114

[17] F. Covito, E. Perfetto, A. Rubio, and G. Stefanucci, Phys. Rev. A 97, 061401 (2018), URL https://link. aps.org/doi/10.1103/PhysRevA.97.061401

[18] S. Kurth, G. Stefanucci, C.-O. Almbladh, A. Rubio, and E. K. U. Gross, Phys. Rev. B 72, 035308 (2005), URL https://link.aps.org/doi/10. 1103/PhysRevB.72.035308

[19] C. Verdozzi, G. Stefanucci, and C.-O. Almbladh, Phys. Rev. Lett. 97, 046603 (2006), URL https://link.aps. org/doi/10.1103/PhysRevLett.97.046603

[20] G. Stefanucci, S. Kurth, A. Rubio, and E. K. U. Gross, Phys. Rev. B 77, 075339 (2008), URL https://link. aps.org/doi/10.1103/PhysRevB.77.075339

[21] P. Myöhänen, A. Stan, G. Stefanucci, and R. van Leeuwen, Phys. Rev. B 80, 115107 (2009), URL https: //link.aps.org/doi/10.1103/PhysRevB.80.115107.

[22] G. Stefanucci, E. Perfetto, and M. Cini, Phys. Rev. B 81, 115446 (2010), URL https://link.aps.org/doi/ 10.1103/PhysRevB.81.115446

[23] M. Hentschel, R. Kienberger, C. Spielmann, G. A. Reider, N. Milosevic, T. Brabec, P. Corkum, U. Heinzmann, M. Drescher, and F. Krausz, Nature 414, 509 (2001), URL http://dx.doi .org/10.1038/35107000.

[24] P. Lipavský, V. Spička, and B. Velický, Phys. Rev. B 34, 6933 (1986), URL https://link.aps.org/doi/10. 1103/PhysRevB.34.6933

[25] C.-O. Almbladh, A. L. Morales, and G. Grossmann, Phys. Rev. B 39, 3489 (1989), URL https://link.aps . org/doi/10.1103/PhysRevB.39.3489

[26] C.-O. Almbladh and A. L. Morales, Phys. Rev. B 39, 3503 (1989), URL https://link.aps.org/doi/10. 1103/PhysRevB.39.3503

[27] E. Perfetto and G. Stefanucci, in preparation (2018).

[28] E. V. Boström, A. Mikkelsen, C. Verdozzi, E. Perfetto, and G. Stefanucci, Nano Lett. 18, 785 (2018), URL https://doi.org/10.1021/acs.nanolett.7b03995

[29] E. Perfetto, D. Sangalli, A. Marini, and G. Stefanucci, The Journal of Physical Chemistry Letters 9, 1353 (2018), URL https://doi.org/10.1021/acs.jpclett. $8 \mathrm{~b} 00025$

[30] G. A. Sawatzky, Phys. Rev. Lett. 39, 504 (1977), URL https://link.aps.org/doi/10.1103/PhysRevLett.39. 504

[31] M. Cini, Solid State Communications 24, 681 (1977), ISSN 0038-1098, URL http://www.sciencedirect.com/ science/article/pii/0038109877903908

[32] L. J. Sham and M. Schlüter, Phys. Rev. Lett. 51, 1888 (1983), URL https://link.aps.org/doi/10. 1103/PhysRevLett.51.1888

[33] R. van Leeuwen, Phys. Rev. Lett. 76, 3610 (1996), URL https://link.aps.org/doi/10.1103/PhysRevLett.76. 3610 